\title{
The effects of hypnotherapy compared to cognitive behavioral therapy in depression: a NIRS-study using an emotional gait paradigm
}

\author{
Alina Haipt ${ }^{2}\left(\mathbb{D} \cdot\right.$ David Rosenbaum $^{2} \cdot$ Kristina Fuhr $^{2} \cdot$ Martin Giese $^{3} \cdot$ Anil Batra $^{1,2} \cdot$ Ann-Christine Ehlis $^{1,2}$
}

Received: 27 May 2021 / Accepted: 19 October 2021 / Published online: 3 February 2022

(c) The Author(s) 2022

\begin{abstract}
Hypnotherapy (HT) is a promising approach to treating depression, but so far, no data are available on the neuronal mechanisms of functional reorganization after HT for depressed patients. Here, 75 patients with mild to moderate depression, who received either HT or Cognitive Behavioral Therapy (CBT), were measured before and after therapy using functional nearinfrared spectroscopy. We investigated the patients' cerebral activation during an emotional human gait paradigm. Further, rumination was included as predictor. Our results showed a decrease of functional connectivity (FC) between two regions that are crucial to emotional processing, the Extrastriate Body Area (EBA) and the Superior Temporal Sulcus (STS). This FC decrease was traced back to an activation change throughout therapy in the right STS, not the EBA and was only found in the HT group, depending on rumination: less ruminating HT patients showed a decrease in right STS activation, while highly ruminating patients showed an increase. We carefully propose that this activation change is due to the promotion of emotional experiences during HT, while in CBT a focus lay on activating behavior and changing negative cognitions. HT seemed to have had differential effects on the patients, depending on their rumination style: The increase of right STS activation in highly ruminating patients might mirror the improvement of impaired emotional processing, whilst the decrease of activation in low ruminating patients might reflect a dismissal of an over-compensation, associated with a hyperactivity before therapy. We conclude that HT affects emotional processing and this effect is moderated by rumination.
\end{abstract}

Keywords Hypnotherapy $\cdot$ Cognitive behavioral therapy $\cdot$ Emotional processing $\cdot$ Temporal lobe $\cdot$ Depression

\section{Introduction}

Depression is a widespread mental disorder currently affecting an estimated number of 264 million people worldwide [1]. Besides somatic and emotional symptoms [2] depression is associated with self-referential thoughts of negative content about the past, future and self, called rumination [3, 4]. Cognitive Behavioral Therapy (CBT) has been shown to be an effective treatment for depressed patients [5]. It

Alina Haipt

alina.haipt@med.uni-tuebingen.de; alinahaipt@gmail.com

1 Tübingen Center for Mental Health (TüCMH), Tuebingen, Germany

2 Department of Psychiatry and Psychotherapy, University Hospital of Tuebingen, Tuebingen, Germany

3 Section for Computational Sensomotorics, Department of Cognitive Neurology, Hertie Institute for Clinical Brain Research Centre for Integrative Neuroscience, University Hospital of Tuebingen, Tuebingen, Germany seems to help normalize depression-specific aberrant prefrontal [6] and amygdala activity [7]. In a meta-analysis on the neurophysiological effects of depression treatment, the authors report effects in frontal areas bilaterally and the lingual gyrus, middle temporal gyrus and middle cingulate cortex left-hemispherically [8]. The effectiveness of CBT in treating depression might originate in an increase of prefrontal functioning, which is associated with cognitive control $[9,10]$. On the other hand, Hypnotherapy (HT), as one of the oldest techniques used in treating mental and somatic disorders [11], displays a promising alternative to CBT in reducing depressive symptoms [12]. Meta-analyses have substantiated the adjuvant effect of hypnotic interventions in treating depressed patients $[13,14]$ and greater effect sizes in symptom reduction when HT is added to CBT compared to sole CBT elements [15]. Fuhr et al. [16] showed in their WIKI-D study that HT is similarly effective to CBT in reducing mild to moderate depression despite their obvious theoretical and conceptual differences. The neurophysiological effects of HT were investigated in patients with irritable 
bowel syndrome [17] and dental phobia [18]; in recent literature, the neurophysiological effects of hypnosis and HT are reviewed and a biopsychosocial model of hypnosis was suggested $[19,20]$. However, so far, the underlying neurobiological processes of HT applied specifically for treating depressed patients have not been investigated.

Depressed patients show altered emotional processing on a behavioral and perceptional level, including aberrant emotional recognition [21, 22], a negativity bias [23, 24], as well as attention biases [25, 26]. Strikingly, most studies on emotional recognition in general and affective perception during depression have been conducted with faces as stimulus material [27]. However, humans are often forced to use other clues than faces, such as posture or gait [28], to infer emotional states in others. Thus, in this study, we focus on the ability of emotional recognition based on human gait.

Neuroanatomically, emotion recognition based on wholebody movements is related to the Extrastriate Body Area (EBA) in the posterior inferior temporal sulcus/middle temporal gyrus [29-32]. Another region of interest (ROI) is the superior temporal sulcus (STS), which is associated with the analysis of biological motion [33-39] and also theory of mind (ToM) processes [39] like understanding the actions [40-43] or mental states of others [44-48]. It was found to be active when presenting subjects with dynamic emotional body expressions [28, 49-51]. Moreover, depression is associated with abnormalities in the superior temporal cortex (including the superior temporal gyrus (STG) and its lateral part, which is often referred to as STS region [52]). In a meta-analysis of functional magnetic resonance imaging (fMRI) studies, the authors found the STG to be one of the regions most consistently involved in depression [53-56].

In the past years, research has focused on investigating connections between cerebral areas (i.e., neural networks) rather than isolated brain regions. Consequently, in this study, we concentrated on the functional co-activation of distinct brain areas-namely the STS and EBA-in terms of functional connectivity (FC). From a theoretical perspective, emotion recognition based on human gait should be reflected in an increased FC between STS and EBA, since both areas are associated with motion recognition as well as emotional processing (STS) and dynamic emotion recognition (EBA), as described above. A coupling of these two areas could reflect a process of the sensory perception of a dynamic emotional body expression (EBA activation) as well as its integration and interpretation (STS activation).

Based on previous own research including a subsample of patients used in this study, it seems that the extent to which depressed patients ruminate influences cerebral activation [57]. Depressed patients who react habitually with rumination to sad mood (trait rumination) showed less FC within the Default-Mode Network (DMN), a major cerebral network that is associated with processes relevant to depression [58-60].

Functional near-infrared spectroscopy (NIRS) is a noninvasive method for optically based functional imaging, offering many advantages including its easy and quick applicability in a noise-free setting and its (relative) tolerance towards movement $[61,62]$, as well as few exclusion criteria. NIRS has been shown to be an apt method to measure activation changes in cerebral networks $[57,58]$.

Based on the theoretical and empirical background outlined above, we focused on the effects of CBT vs. HT on neurophysiological correlates of emotional processing. We also explored potential moderating influences of rumination and additionally analyzed emotion recognition as a behavioral correlate of emotional processing. Therefore, we examined a subsample of depressed patients who participated in the WIKI-D study [16] before and after therapy (CBT or HT). Using a human gait paradigm [28], activation in cortical areas associated with emotional processing and depression (EBA, STS) was elicited and measured with NIRS. Due to the exploratory nature of this study, our main hypothesis was that changes in the STSEBA network associated with emotional processing would occur over the study period and that these changes would differ between CBT and HT treatment. Due to previous own research, we further hypothesized that rumination would moderate this change in connectivity between and among the groups and that the cerebral effects would be mirrored in the patients' behavior.

\section{Methods}

\section{Subjects}

All participants were recruited from 152 patients (intended to treat) of the WIKI-D study [16], all being diagnosed with a unipolar mild to moderate depressive episode by trained clinicians using the Structured Clinical Interview for DSM-IV (SKID-I; [63]). Exclusion criteria for our neurophysiological measurements were pregnancy or nursing a child, severe neurological diseases (e.g., meningitis, epilepsy), untreated hypertension, diabetes, or other coronary diseases as well as social phobia. In total, we included 75 patients (56 female, 19 male; $18-69$ years, $M=39.24$, $S D=14.85)$ participating in 2 NIRS measurements, 1 before and 1 after therapy (Fig. 1). Nearly one third of the patient sample $(n=24)$ showed at least 1 acute comorbid disorder; 25 patients $(n=25)$ took at least 1 antidepressant medication (36\% SSRIs or other including atypical 


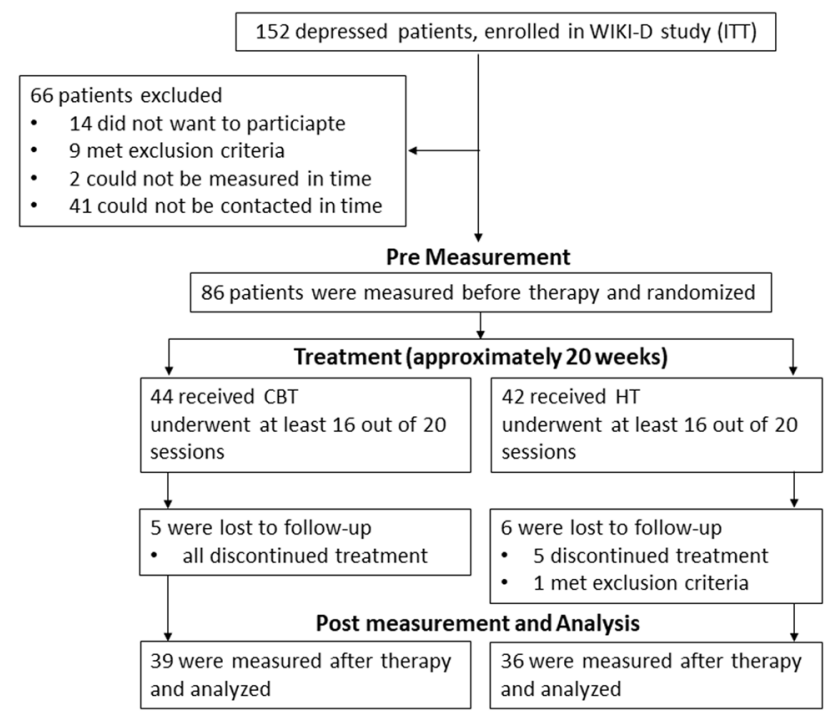

Fig. 1 Procedure of including and measuring patients throughout our study. Number of patients in the WIKI-D study [16] (N); intended to treat (ITT); Cognitive Behavioral Therapy (CBT); Hypnotherapy (HT)

antipsychotics $^{1}$ ), which had to have been taken without changes for 3 months prior to the study. All participants gave their written informed consent to participate in the study.

\section{Stimuli and materials}

To test affective processing, we used an emotional gait paradigm $[28,64]$, portraying sad, happy and neutral stimuli. We measured NIRS data as well as the reaction time and errors on a behavioral level. The happy gait was faster than the sad gait; the neutral walk was presented in three velocities (fast, medium, slow). The displayed video clips ( $3 \mathrm{~s}$ each) showed dark grey volumetric avatars walking across the screen from left to right or vice versa (at an angle of $22^{\circ}$ ). The avatars were uninformative about age, race or sex and still appeared human. The emotional videos (sad and happy) were presented 12 times each, the neutral videos (fast, medium, slow) 4 times each, once for each direction. This resulted in 72 videos total, intermitted by a break. Within blocks, the presentation of the videos was randomized. Rumination as a trait variable was assessed with the Rumination Response Scale [65].

\section{Procedure}

Measurements took place after diagnostics and before undergoing psychotherapy, and after therapy completion (Fig. 1).

\footnotetext{
${ }^{1}$ Besides NaSSA, tricyclic antidepressants, hypericum, SSNRI, agomelatin, bupropion, anticonvulsive medication.
}

Patients were randomly assigned to either CBT $(n=39)$ or HT $(n=36)$. The therapy was considered completed when patients underwent at least 16 of the 20 sessions, which applied to 76 patients. One patient became pregnant during therapy and was excluded from the second measurement. Each NIRS measurement lasted $2 \mathrm{~h}$ and included two additional paradigms besides the gait paradigm, the results of which will be reported elsewhere. Moreover, RRS data were collected between paradigm presentations.

During the measurement, oxygenated $\left(\mathrm{O}_{2} \mathrm{Hb}\right)$ and deoxygenated hemoglobin $(\mathrm{HHb})$ were recorded continuously after a $10 \mathrm{~s}$ baseline measurement. Subjects were asked to judge the portrayed emotion of a walking avatar by pressing the allocated response button (arrow buttons: left, down, and right). The down button always corresponded to neutral gait, the left and right buttons served as response buttons for sad or happy gait (balanced across subjects). Responses could be given as soon as the emotion was recognized. Subjects were not specifically asked to answer as quickly as possible to promote emotional processing over guessing. After a practice trial with feedback, the main experiment began. Each trial started with a fixation cross in the middle of the computer screen (400 ms), followed by a blank screen (100 ms) and an avar video clip (2000 ms), which was followed by $200 \mathrm{~ms}$ blank screen. The duration of the inter-trial interval varied randomly among 5000 and 9000 ms. After 36 trials, the subjects were given a break, which they could end themselves. The experiment lasted about 18-20 min. All subjects received a small monetary compensation for their time.

\section{Near-infrared spectroscopy and regions of interest}

Light in the near-infrared spectrum can penetrate the skull and other biological tissue. Depending on the $\mathrm{O}_{2} \mathrm{Hb}$ and $\mathrm{HHb}$ in the underlying brain tissue, the light is absorbed differently and thus indicates the relative concentration of both chromophores. From this concentration conclusions can be drawn on cortical activation levels underneath the measurement probes. In our study we used an ETG-4000 Optical Topography System (Hitachi Medical Corporation, Tokyo, Japan) with a 52-channel array of 33 optodes (17 light emitters and 16 detectors) covering posterior-occipital and temporal brain areas (temporal resolution: $10 \mathrm{~Hz}$ ). The inter-optode distance was $3 \mathrm{~cm}$ and near-infrared light with two wavelengths (695 and $830 \mathrm{~nm}$ ) was used. The optodes were arranged in a $3 \times 11$-rectangular shape on the subjects' heads in respect to the international 10/20 System [66]. Channel 37 (middle channel in the lowest channel row) was placed over Oz; the anterior channels 43 (left) and 52 (right) were positioned towards the temporal positions T3 and T4, respectively. To assign the channel positions to the anatomy, a neuronavigation system was used on a volunteer's head. 
Fig. 2 Regions of Interest in the right and left cerebral hemisphere. The dashed circles portray the STS region (Channels: 8, 19, 29 (right); 3, 13, 14, 24 (left)), the dotted circles the EBA region (Channels: 39 (right); 35 (left)). Superior Temporal Sulcus (STS); Extrastriate Body Area (EBA)
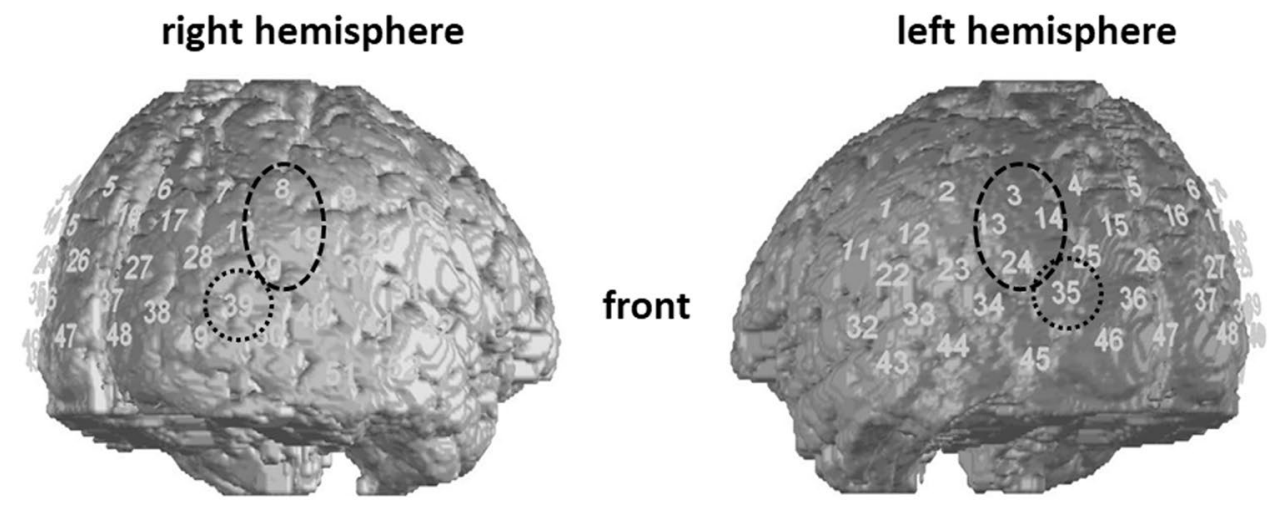

We selected ROIs according to previous findings [28]: namely the EBA and the STS region (including the STG; Fig. 2). Due to limited spatial resolution in NIRS [67, 68], we do not distinguish between activation due to motion perception or emotional perception-both evoking activation in the STS.

\section{Data analyses}

\section{Preprocessing}

The recorded NIRS data were preprocessed and brain plots were generated using MATLAB R2017b (MathWorks Inc, Natick, USA). Preprocessing included applying an algorithm for movement artefact reduction [69]. Then, all signals were visually inspected for local artefacts, $3 \%$ of the channels in the pre data and $4 \%$ in the post data were interpolated from adjacent channels. All signals were then scanned for biting artefacts and contaminated events were excluded from further analysis. We applied a band-pass filter $(0.008-0.25 \mathrm{~Hz})$ to minimize high- and low-frequency noise. Signals were z-transformed to compare between subjects, then the FC between STS and EBA was calculated. To explain the FC effects, post hoc analyses were conducted on the ROIs separately. For this, the NIRS signals were fitted to a model hemodynamic response function in a general linear model based regression $[28,70]$. Based on previous studies using the same paradigm (e.g., [28]) as well as visual inspection of the current data set, peak time was set to $12 \mathrm{~s}$ with a peak dispersion of $2 \mathrm{~s}$. In the behavioral data, responses given in under $300 \mathrm{~ms}$ were excluded because we do not expect them to result from cognitive processing.

\section{Statistics}

For the behavioral and NIRS data analyses, as well as nonbrain figures, we used R Studio (R Studio Inc, Boston, USA). To account for the STS-EBA FC pre therapy and the therapy effect concurrently, we calculated the change score (CS): FC(post therapy)—FC (pre therapy), which served as dependent variable. Linear regression models were constructed consecutively adding fixed effects (first additively, then multiplicatively), in this order: $\mathrm{FC}($ pre), therapy group $(\mathrm{CBT}=0$ vs. $\mathrm{HT}=1)$, rumination (continuous variable) and portrayed emotion (neutral, sad, happy). The models were compared using F-tests. The most complex model accounting for significantly more variance than the previous model was selected. The regression models were constructed in the following way:

$\mathrm{CS}=\beta_{0}+\beta_{i} \times X+\varepsilon$.

We applied the same regression analyses, including the significant predictors, on the behavioral data to capture changes in emotion recognition. We calculated the relative error rate (RER) (sum of errors/trial number) and its CS (RER post therapy-RER pre therapy), which served as dependent variable in regression analyses. Reaction times were looked at on a descriptive level, since they could not be interpreted: no instruction was given to answer as quickly as possible. Post hoc two-tailed t-tests or correlation tests were conducted to further analyze interaction effects. Level of significance was $\alpha=0.05$.

\section{Results}

In the left hemisphere, the linear model containing pre FC as an additive term was the best fitting model for the CS, explaining a significant proportion of variance $\left(F(1,223)=169.20, p<0.001, r^{2}=0.43\right)$. No further predictors contributed significantly. The FC before therapy significantly predicted the $\mathrm{FC}$ change in the left hemisphere $(\beta=-0.78, t(223)=-13.01, p<0.001)$ : The lower the FC between STS and EBA before therapy, the more it increased and vice versa (Online Resource 1). This effect could reflect a regression towards the mean effect, but also regulatory changes over time: patients who showed little EBA-STS coupling at baseline developed this coupling over therapy; patients who had lots of coupling between these two ROIs, 


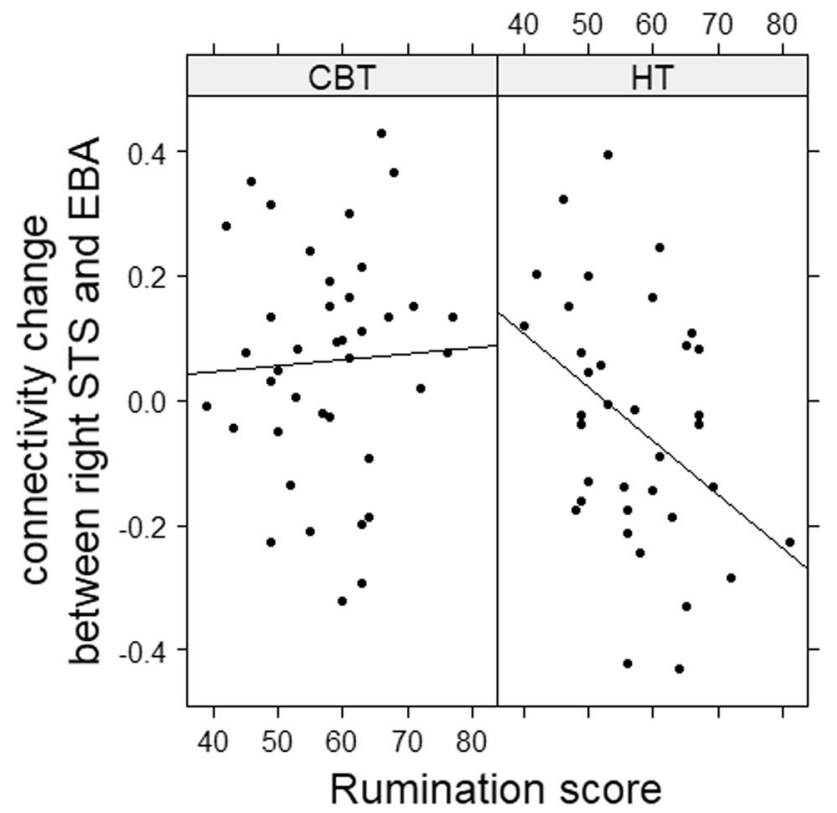

Fig. 3 Correlation between the change of connectivity between the right STS and EBA separately for the two therapy groups. Superior Temporal Sulcus (STS); Extrastriate Body Area (EBA); Cognitive Behavioral Therapy (CBT); Hypnotherapy (HT)

possibly reflecting an overcompensation, showed decreased FC.

For the right hemisphere, the model on the CS including pre $\mathrm{FC}$ as additive term and rumination and group as multiplicative predictors was the most complex model contributing significantly to the explained variance $(F(4$, $\left.220)=42.30, p<0.001, r^{2}=0.42\right)$. This model revealed a significant influence of pre FC $(\beta=-0.70, t(220)=$ $-11.21, p<0.001)$, group $(\beta=0.37, t(220)=2.60$, $p=0.01)$ and an interaction between rumination and group $(\beta=-0.01, t(220)=-3.04, p=0.003)$. The preFC effect implies, again, a negative connection between pre $\mathrm{FC}$ and the CS, thus portraying a regression to the mean on an analytical level and a possible compensation effect on a content level. The main effect for therapy group reflects a change in FC throughout therapy only in the HT group, while the interaction effect further specifies this change within the HT group, depending on rumination. To better understand this interaction effect, we looked at the association between rumination and the CS separately for both groups. In the CBT group, there was a non-significant positive correlation between rumination and the CS $(r(37)=0.05, p=0.77)$, while there was a significant negative correlation $(r(34)=-0.40, p=0.02)$ in the HT group (Fig. 3). Since the predictor "emotion", accounting for the emotional valence of the portrayed gait, did not add significantly to the explanation of variance, the data were
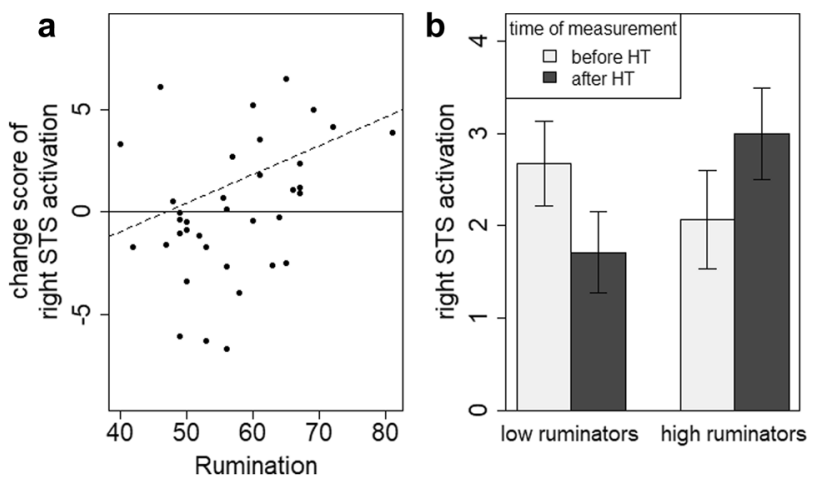

Fig. 4 a CS below and above zero (black line) and the regression line (dashed line) between the CS of right STS activation and rumination, the result of our regression analysis (including preactivation as additive predictor). b Categorial illustration of the association between rumination and the activation of the right STS in the HT group. Change Score (CS; Superior Temporal Sulcus (STS); Hypnotherapy (HT)

pooled across all conditions (happy, sad, and neutral) for further analyses.

To further uncover neurophysiological changes underlying the right hemispheric decrease of FC in the HT group, we conducted post hoc analyses on the activation of the right STS and EBA separately in the HT group. For both ROIs we followed the same regression approach constructing the models consecutively, CS being the dependent variable and adding preactivation and rumination as fixed effects. As assumed, for the right STS the model including preactivation and rumination yielded significance $(F(2,33)=13.84$, $\left.p<0.001, r^{2}=0.42\right)$ and revealed main effects for preactivation $(\beta=-0.64, t(33)=-4.50, p<0.001)$ as well as rumination $(\beta=0.14, t(33)=3.01, p=0.005)$ on the CS (which results in a significant correlation between rumination and CS of $r(34)=0.35, p=0.04)$ indicating an increase in activation for those patients that showed less activation before HT and vice versa, equivalent to the previous analyses. This, again, could show a regression to the mean and possibly a compensation effect. The rumination effect indicates that higher levels of rumination were associated with a greater increase in right STS activation when patients received HT (Fig. 4a). Considering the distribution of CS, it became apparent that negative changes (i.e., a decrease) in right STS activation occurred in a rather large subgroup of patients (who tended to show lower trait rumination scores; Fig. 4a), while positive changes appeared more often in patients that tended to ruminate more. This might suggest a categorical relation between rumination and activation changes rather than a continuous one. For illustration purposes, this categorial relation is portrayed in Figs. $4 \mathrm{~b}$ and 5.

For the activation in the right EBA in the HT group, no model including any predictors contributed to the 
Fig. 5 The effect of rumination on the change of STS activation throughout therapy in the HT group. The figure portrays a t-test between activation before therapy (pre) compared to after therapy (post) (post-pre) in the HT group for either low or high ruminators. Superior Temporal Sulcus (STS); Hypnotherapy (HT)

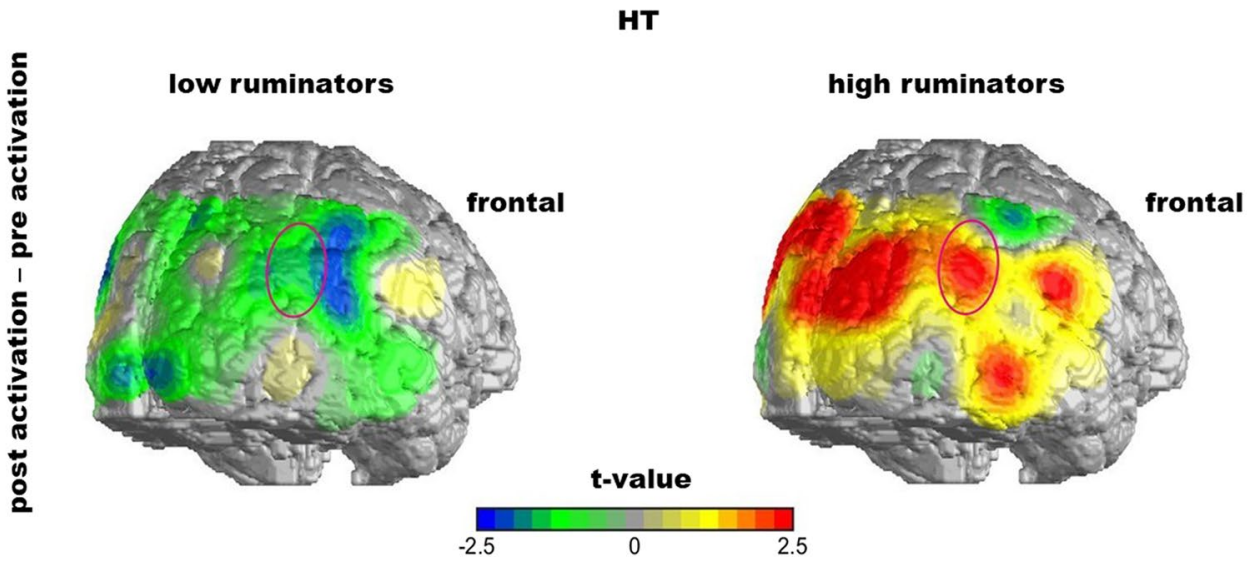

explanation of variance implying no systematic change of activation in the right EBA in the HT group. We conclude that the decrease in right STS-EBA connectivity in the HT group could be derived from a change in activation in the right STS. Further, the STS activation change depended on rumination: while patients with rather low rumination scores showed decreased right STS activation after therapy, this effect was reverse for "high ruminators". Therefore, we explain the decrease in right STS-EBA connectivity with this differential STS activation effect. Next, and for the purpose of completion, we checked for the activation in the right STS before therapy and whether it differentiated between the groups to rule out possible group effects due to different preactivation. We did not find a difference in right STS activation between groups before therapy $(M(\mathrm{HT})=2.24, S D(\mathrm{HT})=3.02 ; M(\mathrm{CBT})=2.53$, $S D(\mathrm{CBT})=3.00 ; t(73)=0.41, p=0.69)$. Finally, as an additional post hoc analysis, we correlated rumination with selfreported symptoms before therapy in both groups and found a significant correlation $(r(73)=0.33, p=0.004)$. This offers an alternative explanation for the HT effect: The effect of HT could originate in initial symptom severity which is linked to rumination.

\section{Behavioral data}

On a descriptive level, reaction times decreased from before to after therapy, and in the HT group there is a slight positive correlation between reaction time and rumination (Online Resource 2). For the RER, descriptive parameters show a decrease over time (as can be seen in the tables, Online Resource 3). Further analysis revealed no systematic connection between RER, rumination, and groups. Applying the same regression method (stepwise regression, excluding the factor "emotion"), the model explaining a significant amount of variance $\left(F(1,65)=92.75, p<0.001, r^{2}=0.58\right)$ included the RER before therapy to be the only predictor of the CS of RER $(\beta=-0.90, t(65)=-9.63, p<0.001)$. This result was consistent, even when 2 outliers were excluded from the analysis $\left(F(1,63)=12.82, p=0.001, r^{2}=0.12\right.$, RER before therapy as predictor: $\beta=-0.52, t(63)=-3.58 p=0.001)$. Thus, patients who either made very few or many mistakes at baseline showed a bigger change in their error rate after therapy (resulting in fewer mistakes for those who had many mistakes at first or more mistakes for those who had few mistakes before therapy). This seems to reflect a typical regression to the mean effect and partly a floor effect since the RER could not sink below zero. Since we did not find group- or rumination-specific effects in the behavioral data, we concluded that the activation change in the right STS in the HT group did not reflect the patients' behavior.

\section{Discussion}

This study addressed the question whether two psychotherapies (CBT and HT) used in depression treatment affect the brain differently. Since the content of CBT and HT differs widely, particularly regarding the work with emotions, we hypothesized that these two therapies differ in their effect on a cerebral network - consisting of the STS and EBA-that is associated with emotional processing. Our main results show a decrease in right hemispheric STS-EBA connectivity in patients who received HT. This decrease in FC can mainly be explained by a change of activation in the STS while the EBA did not change in its activation. This activation change in the right STS in the HT group depended on rumination: while subjects with lower rumination scores showed a decrease in their right STS activation, subjects with higher rumination scores showed an activation increase. These specific neurophysiological changes were not mirrored in behavioral data and were independent from the type of displayed emotion.

These results have multiple implications. First, even though both the STS and EBA were previously found to be active during displayed biological motion $[28,31,33,34$, 
$39,49,71,72]$, here, only the right STS showed activation changes throughout therapy. However, since we focus on the co-activation of the EBA and STS, we cannot make firm conclusions about the activation of the EBA during the gait paradigm by itself. Based on our analysis, EBA activation did not change throughout therapy, suggesting it might not be involved in emotional processes changing in psychotherapy; the STS activation change, however, might be related to these emotional processes. The STS region has shown to be involved in pathological brain activation in depressed patients, when confronted with negative emotional stimuli, with clear lateralization, though [53]. The findings of STS activation in response to perceived body motion were heterogenous in regard to lateralization [28, 33, 34, 39, 72]. Thus, the STS seems to play a role in emotional processing influenced by depression-specific HT; the role of lateralization remains to be investigated.

Interestingly, this STS activation change throughout therapy was only found in the HT group. Since the groups did not differ in right STS activation before therapy, the group effect of the FC change is due to the differential activation after therapy. This difference could be due to the increased focus on emotional therapeutic work in HT compared to CBT. In CBT, the focus lay on activating, analyzing and reviewing behavior and recognizing and changing negative cognitions [73]. This was implemented during therapy sessions and homework. In CBT emotions were mostly addressed on a conversational level. On the other hand, in HT, a focus lay on using the patients' own strengths, positive experiences and memories to create alternatives to negative, wearing thoughts. Namely, pleasant emotions linked to positive personal experiences were induced during trance to make the patients feel competent, hopeful, and strong. The trances were recorded and handed to the patients to listen to them again at home. This might have led to a repetition of the emotional experiences. We argue that HT promoted changing one's perspective since multiple trances focused on putting oneself in a former or later self, possibly reflecting ToM processes. Since the STS region is associated with these emotional and ToM processes [39, 53] it seems fitting that right STS activation changed throughout HT, but not CBT. CBT, on the other hand, has been shown to normalize aberrant amygdala [7] and prefrontal [6] activity, the latter being associated with cognitive control $[9$, $10,74]$.

Interestingly, hypnosis has been shown to lead to decreased prefrontal activity $[89,90]$ and disrupted prefrontal FC [91]. Therefore, the PFC seems to be important to consider when searching for the mechanisms underlying therapeutic effects of HT. Our research group investigated prefrontal areas in the same study sample as presented here (using a different paradigm and NIRS cap placement); the results will be published elsewhere.
Secondly, we found that the right STS activation change after HT depended on the patients' rumination: Patients who tended to ruminate less before therapy showed a decrease while patients who tended to ruminate more showed an increase in right STS activation after HT suggesting a categorical division of patients in high and low ruminators. This is in line with previous research of our group, in which differential DMN activity was found for high versus low ruminating depressed patients [57]. In previous research, trait rumination was shown to be positively associated with depressive symptoms and their severity [76-77], mediating risk factors for depression [78], and predicting treatment success [79]. We, as well, found a correlation between rumination and self-reported symptoms. Further research has shown abnormal functioning of the DMN in depressed subjects [60] and decreased FC within the DMN in depressed subjects who tended to ruminate [57]. The DMN also seems to be linked to ToM and thus emotional processes, which elicit increased activity in lateral regions like the STS and increased coupling with the medial prefrontal cortex, a core node of the DMN [80]. Furthermore, multiple authors suggested a link between hypnotic trance and the DMN e.g., [77-80]. More detailed results on the connection between $\mathrm{HT}$ and the DMN will be derived from a resting-state measurement also conducted in the context of this study and published elsewhere. In line with this research, our results might indicate a greater degree of depression in highly ruminating patients whose decreased STS activity before therapy possibly reflected impaired emotional processing. The increase of STS activity throughout HT in these patients could be a neurophysiological correlate of normalized emotional processing induced by HT as described above. On the other hand, low ruminating depressed patients showed a decrease in STS activity throughout HT. Our results propose that low ruminators show less severe depressive symptoms and might therefore be less impaired in emotional processing. Opposed to the more severely impaired patients, the less impaired might still be capable to compensate abnormal emotional processing with a certain effort. This compensation effort could be associated with hyperactivity in the right STS. The benefit of HT for these patients could be linked to a decrease in right STS activity due to the reduction of over-compensation. Further research is needed to underpin this hypothesis and clarify the interaction of rumination and symptom severity on depression-related cerebral activity.

Thirdly, the absence of significant predictive value of the factors group and rumination for the error rate (RER) implies that activity changes in the right STS did not influence the behavioral data in a measurable way. A possible explanation is that the RER is based on the reaction as a consequence of emotional recognition. This reaction is rather a judgement than a mirror of the preceding emotional processing. Emotional judgement, though, is associated with 
prefrontal activity [85-87] and might not be correlated with temporal activity. To get to the core of this effect, further research with more specific hypotheses is needed.

Lastly, the portrayed emotion did not predict the FC change between EBA and STS throughout therapy nor did it predict the activity change in the right STS. In previous studies, differences in EBA activation occurred between negative emotional body expressions compared to neutral expressions [28, 49, 71, 72], but all of these studies were conducted with healthy subjects. Indeed, depressed subjects have shown aberrant emotional processing on a behavioral level compared to healthy controls [22-24, 26, 88], but in these studies cerebral activation was not assessed. The cerebral processes measured in our study and the changes throughout HT do not seem to be emotion-specific.

\section{Study limitations and future research}

To our knowledge, no previous study has investigated the FC of these two regions even though they were found to be involved in the processing of dynamic, emotional body expressions, and none of them including depressed subjects $[28,49,71]$. This present study is of an exploratory nature and consequently more research is needed to investigate the FC between STS and EBA in depressed subjects. Moreover, other regions involved in the processing of emotional dynamic body expression, like the amygdala [49], cannot be measured with NIRS. Furthermore, due its relatively low spatial resolution, NIRS might not be an adequate method to distinguish motion perception and emotional perception, which are both associated with activation in the STG. e.g., $[35,36]$. Therefore, interpretations about emotional processing associated with STS activity need to be considered carefully. To further investigate the co-activation of other areas, including subcortical ones, and to distinguish intra-STS areas, fMRI data should be obtained. Another methodological restriction is that we could not perform a short-channel regression since our NIRS system does not allow to routinely include short-distance channels. Therefore, we cannot exclude the possibility that superficial perfusion changes contributed to our NIRS data. A further limitation is that systemic effects were not controlled during data preprocessing which might have influenced the results.

The assumption that HT fosters more emotional processes than CBT was based on manuals used in the WIKI-D study [16] and interviews with experts. We do not claim this to be the only differentiating factor between HT and CBT; further research is required to clarify the differences. In the future, rumination should be continuously included when investigating depression as it seems to be linked to abnormal, depression-specific, cerebral functioning [57]. This would help to better understand depression in its many facets and to individualize treatment.

\section{Conclusion}

This first of its kind study explores the cerebral processes underlying differential effects of Hypnotherapy (HT) and Cognitive Behavioral Therapy (CBT) in treating depressed patients. In the treatment of depressed patients, HT was not inferior to CBT [16]. Still, how these treatments work (or which group pf patients responds well to either type of therapy) remained unclear. In our study, we investigated the effects of HT and CBT on cerebral activation associated with emotional processing. We found that activation in the right STS changed throughout therapy specifically in the HT group. Further, rumination played a crucial role in predicting activation changes in the HT group and thus seems to be an important factor to consider when drawing conclusions about depression treatment and differential indication. We conclude that HT affects emotional processing in depressed patients and this effect is moderated by the patients' rumination style. Depression-specific HT, its content, effects and underlying processes have rarely been investigated and therefore many of our assumptions were exploratory in nature. This paper was designed to be a first step in the direction of exploring HT and its differential effects.

Supplementary Information The online version contains supplementary material available at https://doi.org/10.1007/s00406-021-01348-7.

Acknowledgements We thank the staff of the Department of Psychophysiology and Optical Imaging of the University Hospital of Tuebingen, namely Ramona Taeglich and Betti Schopp, Florens Goldbeck, Moritz Maier, Sarah Weber and Hendrik Laicher, for their time and work measuring the patients.

Authors' contributions AH: Term, conceptualization, methodology, formal analysis, investigation, writing-original draft, visualization. DR: Conceptualization, software, data curation, writing - review and editing. KF: Resources, writing — review and editing, project administration. MG: Methodology, software, writing - review and editing. AB: Supervision, funding acquisition, writing - review and editing. A-CE: Term, conceptualization, methodology, formal analysis, resources, validation, supervision, project administration, funding acquisition, writing-review and editing.

Funding Open Access funding enabled and organized by Projekt DEAL. This study was financially supported by the Milton-Erickson Society for Clinical Hypnosis, Germany (M.E.G.). Funding was used to grant a small, monetary time compensation to all subjects and a part-time wage for the study coordinator. The funding source did not have any further involvement in the study.

Data availability The datasets from the current study are available upon request.

Code availability The codes of the current study are available upon request. 


\section{Declarations}

Conflict of interests The authors declare that they have no conflict of interest.

Ethics approval This study was approved with a positive ethics vote by the Ethics Committee of the University Hospital of Tuebingen (061/2015B02).

Consent to participate All participants gave their written informed consent to participate in the study.

Open Access This article is licensed under a Creative Commons Attribution 4.0 International License, which permits use, sharing, adaptation, distribution and reproduction in any medium or format, as long as you give appropriate credit to the original author(s) and the source, provide a link to the Creative Commons licence, and indicate if changes were made. The images or other third party material in this article are included in the article's Creative Commons licence, unless indicated otherwise in a credit line to the material. If material is not included in the article's Creative Commons licence and your intended use is not permitted by statutory regulation or exceeds the permitted use, you will need to obtain permission directly from the copyright holder. To view a copy of this licence, visit http://creativecommons.org/licenses/by/4.0/.

\section{References}

1. WHO. Depression. 2020. https://www.who.int/news-room/factsheets/detail/depression

2. American Pschological Association (2013) Diagnostic and statistical manual of mental disorders, 5th edn. American Psychological Association, Arlington

3. Nejad AB, Fossati P, Lemogne C (2013) Self-referential processing, rumination, and cortical midline structures in major depression. Front Hum Neurosci 7:1-9. https://doi.org/10.3389/fnhum. 2013.00666

4. Nolen-Hoeksema S (1991) Responses to depression and their effects on the duration of depressive episodes. J Abnorm Psychol 100:569-582. https://doi.org/10.1037/0021-843X.100.4.569

5. Cuijpers P, Berking M, Andersson G, Quigley L, Kleiboer A, Dobson KS (2013) A meta-analysis of cognitive-behavioural therapy for adult depression, alone and in comparison with other treatments. Can J Psychiatry 58:376-385. https://doi.org/10.1177/ 070674371305800702

6. Goldapple K, Segal Z, Garson C, Lau M, Bieling P, Kennedy $S$ et al (2004) Modulation of cortical-limbic pathways in major depression. Arch Gen Psychiatry 61:34-41

7. DeRubeis RJ, Siegle GJ, Hollon SD (2008) Cognitive therapy versus medication for depression: treatment outcomes and neural mechanisms. Nat Rev Neurosci 9:788-796. https://doi.org/10. 1038/nrn2345

8. Boccia M, Piccardi L, Guariglia P (2016) How treatment affects the brain: meta-analysis evidence of neural substrates underpinning drug therapy and psychotherapy in major depression. Brain Imaging Behav 10:619-627. https://doi.org/10.1007/ s11682-015-9429-X

9. Rosenbaum D, Leehr EJ, Rubel J, Maier MJ, Pagliaro V, Deutsch $\mathrm{K}$ et al (2020) Cortical oxygenation during exposure therapy-in situ fNIRS measurements in arachnophobia. NeuroImage Clin 26:102219. https://doi.org/10.1016/j.nicl.2020.102219
10. Karlsson H (2011) How psychotherapy changes the brain. Psychiatr Times 28:1-5

11. Revenstorf D, Peter B (2009) Hypnose in psychotherapie, psychosomatik und medizin. Springer, Heidelberg

12. Kirsch I, Low CB (2013) Suggestion in the treatment of depression. Am J Clin Hypn 55:221-229. https://doi.org/10.1080/00029 157.2012.738613

13. Milling LS, Valentine KE, McCarley HS, LoStimolo LM (2018) A meta-analysis of hypnotic interventions for depression symptoms: high hopes for hypnosis? Am J Clin Hypn 61:227-243. https://doi. org/10.1080/00029157.2018.1489777

14. Shih M, Yang YH, Koo M (2009) A Meta-analysis of hypnosis in the treatment of depressive symptoms: a brief communication. Int J Clin Exp Hypn 57:431-442. https://doi.org/10.1080/00207 140903099039

15. Alladin A, Alibhai A (2007) Cognitive hypnotherapy for depression: an empirical investigation. Int J Clin Exp Hypn 55:147166. https://doi.org/10.1080/00207140601177897

16. Fuhr K, Meisner C, Broch A, Cyrny B, Hinkel J, Jaberg J et al (2021) Efficacy of hypnotherapy compared to cognitive behavioral therapy for mild to moderate depression-results of a randomized controlled rater-blind clinical trial. J Affect Disord 286:166-173. https://doi.org/10.1016/j.jad.2021.02.069

17. Lowén MBO, Mayer EA, Sjöberg M, Tillisch K, Naliboff B, Labus J et al (2013) Effect of hypnotherapy and educational intervention on brain response to visceral stimulus in the irritable bowel syndrome. Aliment Pharmacol Ther 37:1184-97. https://doi.org/10.1111/apt.12319

18. Halsband U, Wolf TG (2015) Functional changes in brain activity after hypnosis in patients with dental phobia. J Physiol 109:131-142. https://doi.org/10.1016/j.jphysparis.2016.10.001

19. Halsband U, Wolf TG (2021) Current neuroscientific research database findings of brain activity changes after hypnosis. Am J Clin Hypn 63:372-388. https://doi.org/10.1080/00029157.2020. 1863185

20. Jensen MP, Adachi T, Tomé-Pires C, Lee J, Osman ZJ, Miró J (2015) Mechanisms of hypnosis: toward the development of a biopsychosocial model. Int J Clin Exp Hypn 63:34-75. https:// doi.org/10.1080/00207144.2014.961875

21. Anderson IM, Shippen C, Juhasz G, Chase D, Thomas E, Downey D et al (2011) State-dependent alteration in face emotion recognition in depression. Br J Psychiatry 198:302-308. https://doi.org/10.1192/bjp.bp.110.078139

22. Surguladze SA, Senior C, Young AW, Brébion G, Travis MJ, Phillips ML (2004) Recognition accuracy and response bias to happy and sad facial expressions in patients with major depression. Neuropsychology 18:212-218. https://doi.org/10.1037/ 0894-4105.18.2.212

23. Milders M, Bell S, Platt J, Serrano R, Runcie O (2010) Stable expression recognition abnormalities in unipolar depression. Psychiatry Res 179:38-42. https://doi.org/10.1016/j.psychres. 2009.05.015

24. Gur RC, Erwin RJ, Gur RE, Zwil AS, Heimberg C, Kraemer HC (1992) Facial emotion depression discrimination: II. Behavioral findings in depression. Psychiatry Res 42:241-251

25. Gotlib IH, Krasnoperova E (2004) Attentional biases for negative interpersonal stimuli in clinical depression. J Abnorm Psychol 113:127-135. https://doi.org/10.1037/0021-843X.113.1. 127

26. Suslow T, Dannlowski U, Lalee-mentzel J, Donges U, Arolt V, Kersting A (2004) Spatial processing of facial emotion in patients with unipolar depression : a longitudinal study. J Affect Disord 83:59-63. https://doi.org/10.1016/j.jad.2004.03.003

27. De GB (2009) Why bodies? Twelve reasons for including bodily expressions in affective neuroscience. Philos Trans R Soc B Biol Sci 364:3475-3484. https://doi.org/10.1098/rstb.2009.0190 
28. Schneider S, Christensen A, Häußinger FB, Fallgatter AJ, Giese MA, Ehlis AC (2014) Show me how you walk and I tell you how you feel-a functional near-infrared spectroscopy study on emotion perception based on human gait. Neuroimage 85:380-390. https://doi.org/10.1016/j.neuroimage.2013.07.078

29. Taylor JC, Downing PE (2011) Division of labor between lateral and ventral extrastriate representations of faces, bodies, and objects. J Cogn Neurosci 23:4122-4137. https://doi.org/ 10.1162/jocn_a_00091

30. Downing PE, Jiang Y, Shuman M, Kanwisher N (2001) A cortical area specialized for visual processing of the human body. Science 293:2470-3. https://doi.org/10.1167/1.3.341

31. Lamm C, Decety J (2008) Is the extrastriate body area (EBA) sensitive to the perception of pain in others? Cereb Cortex 18:2369-2373. https://doi.org/10.1093/cercor/bhn006

32. van de Riet W, Grèzes J, de Gelder B (2009) Specific and common brain regions involved in the perception of faces and bodies and the representation of their emotional expressions. Soc Neurosci 4:101-120. https://doi.org/10.1080/17470910701865367

33. Bonda E, Petrides M, Ostry D, Evans A (1996) c-Jun N-terminal kinase activity supports multiple phases of 3D-mammary epithelial acinus formation. J Neurosci 16:3737-3744. https://doi.org/ $10.1387 / \mathrm{ijdb} .113374 \mathrm{sm}$

34. Allison T, Puce A, McCarthy G (2000) Social perception from visual cues: role of the STS region. Trends Cogn Sci 4:267-278. https://doi.org/10.1016/S1364-6613(00)01501-1

35. Grossman E, Donnelly M, Price R, Pickens D, Morgan V, Neighbor $\mathrm{G}$ et al (2000) Brain areas involved in perception of biological motion. J Cogn Neurosci 12:711-20

36. Grossman ED, Blake R (2002) Brain areas active during visual perception of biological motion. Neuron 35:1167-1175. https:// doi.org/10.4324/9780203496190

37. Pelphrey K, Mitchell T, McKeown MJ, Goldstein J, Allison T, McCarthy G (2003) Brain activity evoked by the perception of human walking: controlling for meaningful coherent motion. J Neurosci 23:6819-6825

38. Pelphrey KA, Morris JP, Michelich CR, Allison T, McCarthy G (2005) Functional anatomy of biological motion perception in posterior temporal cortex: an fMRI study of eye, mouth and hand movements. Cereb Cortex 15:1866-1876. https://doi.org/10.1093/ cercor/bhi064

39. Deen B, Koldewyn K, Kanwisher N, Saxe R (2015) Functional organization of social perception and cognition in the superior temporal sulcus. Cereb Cortex 25:4596-4609. https://doi.org/10. 1093/cercor/bhv111

40. Pelphrey KA, Singerman JD, Allison T, McCarthy G (2003) Brain activation evoked by perception of gaze shifts: the influence of context. Neuropsychologia 41:156-170. https://doi.org/10.1016/ S0028-3932(02)00146-X

41. Pelphrey K, Morris J, McCarthy G (2004) Grasping the intentions of others: the perceived intentionality of an action influences activity in the superior temporal sulcus during social perception. J Cogn Neurosci 16:1706-1716

42. Brass M, Schmitt RM, Spengler S, Gergely G (2007) Investigating action understanding: inferential processes versus action simulation. Curr Biol 17:2117-2121. https://doi.org/10.1016/j.cub.2007. 11.057

43. Vander Wyk BC, Hudac CM, Carter EJ, Sobel DM, Pelphrey KA (2009) Action understanding in the superior temporal sulcus region. Psychol Sci 20:771-777

44. Fletcher PC, Happe F, Frith U, Baker SC, Dolan RJ, Frackowiak RSJ et al (1995) Other minds in the brain: a functional imaging study of "theory of mind" in story comprehension. Cognition 57:109-128. https://doi.org/10.1016/0010-0277(95)00692-R

45. Gallagher HL, Happé F, Brunswick N, Fletcher PC, Frith U, Frith CD (2000) Spatial selectivity in the temporoparietal junction, inferior frontal sulcus, and inferior parietal lobule. Neuropsychologia 38:11-21. https://doi.org/10.1167/15.13.15

46. Saxe R, Kanwisher N (2003) People thinking about thinking people: The role of the temporo-parietal junction in "theory of mind." Neuroimage 19:1835-1842. https://doi.org/10.4324/9780203496 190

47. Ciaramidaro A, Adenzato M, Enrici I, Erk S, Pia L, Bara BG et al (2007) The intentional network: how the brain reads varieties of intentions. Neuropsychologia 45:3105-3113. https://doi.org/10. 1016/j.neuropsychologia.2007.05.011

48. Saxe R, Powell LJ (2006) It's the thought that counts. Psychol Sci 17:692-699. https://doi.org/10.1111/J.1467-9280.2006.01768.X

49. Grèzes J, Pichon S, de Gelder B (2007) Perceiving fear in dynamic body expressions. Neuroimage 35:959-967. https://doi.org/10. 1016/j.neuroimage.2006.11.030

50. Kret ME, Pichon S, Grèzes J, De Gelder B (2011) Similarities and differences in perceiving threat from dynamic faces and bodies, An fMRI study. Neuroimage 54:1755-1762. https://doi. org/10.1016/j.neuroimage.2010.08.012

51. Peelen MV, Atkinson AP, Vuilleumier P (2010) Supramodal representations of perceived emotions in the human brain. $\mathrm{J}$ Neurosci 30:10127-10134. https://doi.org/10.1523/JNEUR OSCI.2161-10.2010

52. Takahashi T, Yücel M, Lorenzetti V, Walterfang M, Kawasaki Y, Whittle $S$ et al (2010) An MRI study of the superior temporal subregions in patients with current and past major depression. Prog Neuropsychopharmacol Biol Psychiatry 34:98-103. https://doi.org/10.1016/j.pnpbp.2009.10.005

53. Fitzgerald PB, Laird AR, Maller J, Daskalakis ZJ (2008) A meta-analytic study of changes in brain activation in depression. Hum Brain Mapp 29:683-695. https://doi.org/10.1002/ hbm.20426.A

54. Canli T, Sivers H, Thomason ME, Whitfield-Gabrieli S, Gabrieli JDE, Gotlib IH (2004) Brain activation to emotional words in depressed vs healthy subjects. NeuroReport 15:2585-2588. https://doi.org/10.1097/00001756-200412030-00005

55. Shah PJ, Glabus MF, Goodwin GM, Ebmeier KP (2002) Chronic, treatment-resistant depression and right fronto-striatal atrophy. $\mathrm{Br}$ J Psychiatry 180:434-440. https://doi.org/10.1192/bjp.180.5.434

56. Cullen KR, Gee DG, Klimes-Dougan B, Gabbay V, Hulvershorn L, Mueller BA et al (2009) A preliminary study of functional connectivity in comorbid adolescent depression. Neurosci Lett 460:227-231. https://doi.org/10.1016/j.neulet.2009.05.022

57. Rosenbaum D, Haipt A, Fuhr K, Haeussinger FB, Metzger FG, Nuerk HC et al (2017) Aberrant functional connectivity in depression as an index of state and trait rumination. Sci Rep 7:1-12. https://doi.org/10.1038/s41598-017-02277-z

58. Rosenbaum D, Hagen K, Deppermann S, Kroczek AM, Haeussinger FB, Heinzel S et al (2016) State-dependent altered connectivity in late-life depression: a functional near-infrared spectroscopy study. Neurobiol Aging 39:57-68. https://doi.org/ 10.1016/j.neurobiolaging.2015.11.022

59. Menon V (2011) Large-scale brain networks and psychopathology: a unifying triple network model. Trends Cogn Sci 15:483506. https://doi.org/10.1016/j.tics.2011.08.003

60. Greicius MD, Flores BH, Menon V, Glover GH, Solvason HB, Kenna $\mathrm{H}$ et al (2007) Resting-state functional connectivity in major depression: abnormally increased contributions from subgenual cingulate cortex and thalamus. Biol Psychiatry 62:429437. https://doi.org/10.1016/j.biopsych.2006.09.020

61. Ernst LH, Schneider S, Ehlis AC, Fallgatter AJ (2012) Functional near infrared spectroscopy in psychiatry: a critical review. J Near Infrared Spectrosc 20:93-105. https://doi.org/10.1255/jnirs. 970

62. Fallgatter AJ, Ehlis AC, Wagener A, Michel T, Herrmann MJ (2004) Nah-Infrarot-Spektroskopie in der Psychiatrie. Nervenarzt 75:911-916. https://doi.org/10.1007/s00115-002-1457-2 
63. Wittchen H-U, Wunderlich U, Gruschwitz S, Zaudig M. SKID I. (1997) Strukturiertes Klinisches Interview für DSM-IV. Achse I: Psychische Störungen. Göttingen: Hogrefe

64. Roether CL, Omlor L, Christensen A, Giese MA (2009) Critical features for the perception of emotion from gait. J Vis 9:1-32

65. Nolen-Hoeksema S, Morrow J (1991) A prospective study of depression and posttraumatic stress symptoms after a natrual disaster: the 1989 Loma Prieta earthquake. J Pers Soc Psychol 61:115-121. https://doi.org/10.1037/0022-3514.61.1.115

66. Jasper HH (1958) The ten-twenty electrode system of the International Federation. Electroencephalogr Clin Neurophysiol 10:371-375

67. McCormick PW, Stewart M, Lewis G, Dujovny M, Ausman JI (1992) Intracerebral penetration of infrared light, Technical note. J Neurosurg 76:315-318

68. Wabnitz H, Moeller M, Liebert A, Obrig H, Steinbrink J, MacDonald R (2010) Time-resolved near-infrared spectroscopy and imaging of the adult human brain. In: Takahashi E, Bruley D (eds) Oxygen transport to tissue XXXI, vol 662. Springer, Boston, pp 143-148

69. Cui X, Bray S, Reiss A (2010) Functional near infrared spectroscopy (NIRS) signal improvement based on negative correlation between oxygenated and deoxygenated hemoglobin. Neuroimage 49:3039-3046

70. Plichta MM, Heinzel S, Ehlis AC, Pauli P, Fallgatter AJ (2007) Model-based analysis of rapid event-related functional near-infrared spectroscopy (NIRS) data: a parametric validation study. Neuroimage 35:625-634. https://doi.org/10.1016/j.neuroimage.2006. 11.028

71. Pichon S, de Gelder B, Grèzes J (2008) Emotional modulation of visual and motor areas by dynamic body expressions of anger. Soc Neurosci 3:199-212. https://doi.org/10.1080/17470910701394368

72. Sinke CBA, Sorger B, Goebel R, de Gelder B (2010) Tease or threat? Judging social interactions from bodily expressions. Neuroimage 49:1717-1727. https://doi.org/10.1016/j.neuroimage. 2009.09.065

73. Wilhelm-Goessling C, Schweizer C, Dürr C, Fuhr K, Revenstorf D (2020) Hypnotherapie bei Depressionen: Ein Manual für Psychotherapeuten, 1st edn. Kohlhammer Verlag, Stuttgart

74. Rosenbaum D, Maier MJ, Hudak J, Metzger FG, Wells A, Fallgatter AJ et al (2018) Neurophysiological correlates of the attention training technique: a component study. NeuroImage Clin 19:1018-1024. https://doi.org/10.1016/j.nicl.2018.06.021

75. Koval P, Kuppens P, Allen NB, Sheeber L (2012) Getting stuck in depression: the roles of rumination and emotional inertia. Cogn Emot 26:1412-1427. https://doi.org/10.1080/02699931.2012. 667392

76. Smith JM, Alloy LB (2009) A roadmap to rumination: a review of the definition, assessment, and conceptualization of this multifaceted construct. Clin Psychol Rev 29:116-128. https://doi.org/ 10.1016/j.cpr.2008.10.003

77. Ito T, Takenaka K, Tomita T, Agari I (2006) Comparison of ruminative responses with negative rumination as a vulnerability factor for depression. Psychol Rep. 99:763-772. https://doi.org/10.2466/ PR0.99.3.763-772

78. Spasojević J, Alloy LB (2001) Rumination as a common mechanism relating depressive risk factors to depression. Emotion 1:25-37. https://doi.org/10.1037/1528-3542.1.1.25
79. Teismann T, Willutzki U, Michalak J, Schulte D (2008) Bedeutung von Rumination und Ablenkung für den Therapieerfolg depressiver Patienten. Verhaltenstherapie 18:215-222. https://doi.org/ $10.1159 / 000165687$

80. Spreng RN, Grady CL (2010) Patterns of brain activity supporting autobiographical memory, prospection, and theory of mind, and their relationship to the default mode network. J Cogn Neurosci 22:1112-1123. https://doi.org/10.1162/jocn.2009.21282

81. Pyka M, Burgmer M, Lenzen T, Pioch R, Dannlowski U, Pfleiderer B et al (2011) Brain correlates of hypnotic paralysis-a resting-state fMRI study. Neuroimage 56:2173-2182

82. Cojan Y, Waber L, Schwartz S, Rossier L, Forster A, Vuilleumier $P$ (2009) The brain under self-control: modulation of inhibitory and monitoring cortical networks during hypnotic paralysis. Neuron 62:862-875. https://doi.org/10.1016/j.neuron.2009.05.021

83. Deeley Q, Oakley D, Toone B, Giampietro V, Brammer MJ, Williams SCR et al (2012) Modulating the default mode network using hypnosis. Int J Clin Exp Hypn 60:206-228. https://doi.org/ 10.1080/00207144.2012.648070

84. McGeown WJ, Mazzoni G, Venneri A, Kirsch I (2009) Hypnotic induction decreases anterior default mode activity. Conscious Cogn 18:848-855. https://doi.org/10.1016/j.concog.2009.09.001

85. Grimm S, Beck J, Schuepbach D, Hell D, Boesiger P, Bermpohl $F$ et al (2008) Imbalance between left and right dorsolateral prefrontal cortex in major depression is linked to negative emotional Judgment : an fMRI study in severe major depressive disorder. Biol Psychiatry 63:369-376. https://doi.org/10.1016/j.biopsych. 2007.05.033

86. Grimm S, Schmidt CF, Bermpohl F, Heinzel A, Dahlem Y, Wyss $M$ et al (2006) Segregated neural representation of distinct emotion dimensions in the prefrontal cortex-an fMRI study. Neuroimage 30:325-340. https://doi.org/10.1016/j.neuroimage.2005.09. 006

87. Northoff G, Duncan NW, Hayes DJ (2010) The brain and its resting state activity-experimental and methodological implications. Prog Neurobiol 92:593-600. https://doi.org/10.1016/j.pneurobio. 2010.09.002

88. Gotlib IH, Kasch KL, Traill S, Joormann J, Arnow BA, Johnson SL (2004) Coherence and specificity of information-processing biases in depression and social phobia. J Abnorm Psychol 113:386-98. https://doi.org/10.1037/0021-843X.113.3.386

89. Gruzelier JH (2002) A review of the impact of hypnosis, relaxation, guided imagery and individual differences on aspects of immunity and health. Stress 5:147-163. https://doi.org/10.1080/ 10253890290027877

90. Jiang H, White MP, Greicius MD, Waelde LC, Spiegel D (2017) Brain activity and functional connectivity associated with hypnosis. Cereb Cortex 27:4083-4093. https://doi.org/10.1093/cercor/ bhw 220

91. Fingelkurts AA, Fingelkurts AA, Kallio S, Revonsuo A (2007) Cortex functional connectivity as a neurophysiological correlate of hypnosis: an EEG case study. Neuropsychologia 45:1452-1462. https://doi.org/10.1016/j.neuropsychologia.2006.11.018 